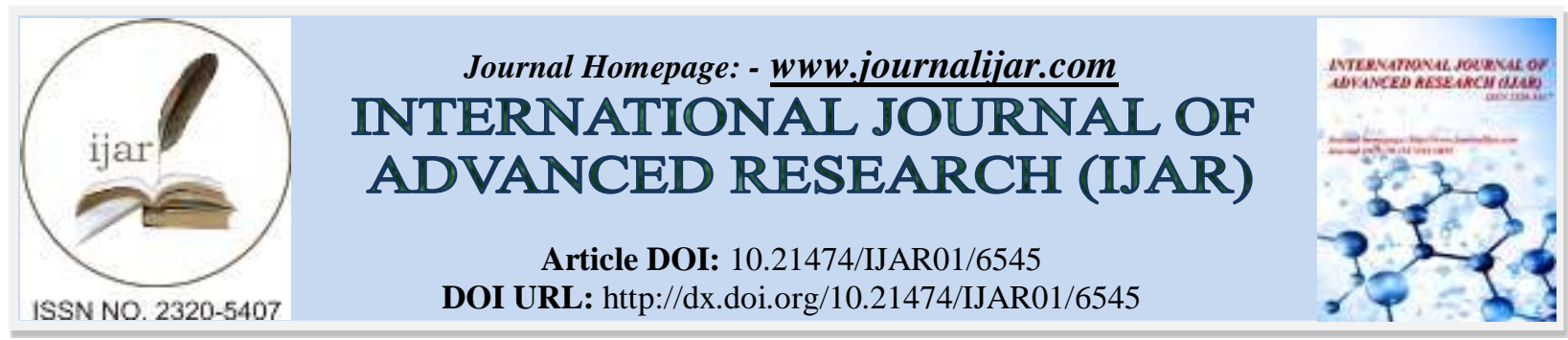

RESEARCH ARTICLE

\title{
INSILICO CHARACTERIZATION OF GLUTATHIONE S-TRANSFERASES AND THEIR INTERACTION STUDY IN MULBERRY SILKWORM, BOMBYX MORI.
}

\author{
Sumathy R, Sivaprasad V and "Manthira Moorthy S. \\ Bioinformatics Center, Central Sericultural Research and Training Institute, Srirampura, Mysuru -570008, \\ Karnataka.
}

\section{Manuscript Info \\ Manuscript History \\ Received: 15 December 2017 \\ Final Accepted: 17 January 2018 \\ Published: February 2018 \\ Key words:- \\ Silkworm, Glutathione S- \\ transferases, Functional annotation, \\ Interaction Studies.}

\begin{abstract}
The Glutathione $\mathbf{S}$-transferases are cytosolic enzymes that are found in both prokaryotes and eukaryotes. The GST multigene family are phase II detoxification enzymes that mainly helps to detoxify the large range of xenobiotic compounds in many organisms. These enzymes play a major role in detoxification pathway along with their related genes. We characterized the each GSTs class of silkworm such as Delta, Epsilon, Omega, Theta, Zeta and the phylogentic tree of GSTs were consructed. Further, we identified the conserved domains of GSTs such as thioredoxin_like and GST_C_family superfamily. Also, the G-site, Hsite and dimer inferace of GSTs were identified. Around 10 conserved motifs were found by analyzing all GSTs of the silkworm. The silkworm genome sequences were mapped into the kegg pathway database and found the silkworm genes that are found in the xenobiotics metabolism pathway. Additionally, 128 protein-protein interactions of silkworm were found from the GSTs and their related genes of metabolic pathways. Further, functional enrichment analysis was shown. This study paves ways to understand the phase II detoxification enzymes, glutathione $\mathbf{S}$-transferases and xenobiotics detoxification metabolic pathway of the mulberry silkworm.
\end{abstract}

Copy Right, IJAR, 2018,. All rights reserved.

\section{Introduction:-}

Glutathione S-transferases (GSTs) are multifunctional superfamily found widely in all organisms. It plays an important role in cellular physiological activities such as detoxifying insecticides, a, b-unstaturated carboyl compounds, xenobiotic compounds, arene oxide, quinines and biosynthesis of hormones (Motoyama and Dauterman, 1980; Clark and Shamaan, 1984; Vontas et al., 2001; Hayes et al., 2005). GSTs are ubiquitous enzymes, conjugating hydrophobic, electrophilic compounds with glutathione by covalent binding and also function against oxidative injury (Singh et al., 2001).

According to the GSTs cellular location, it has been categorized into microsomal, mitochondrial and cystolic GSTs. So far only microsomal and cystolic GSTs have been determined in the insects. Mitochondrial GSTs are found in mammalian peroximes and mitochondria, but are not present in insects (Lander et al., 2004; Morel et al., 2004). Mostly microsomal GSTs comprises of 150 amino acid residues and belongs to memberane associated proteins in eicosanoid and glutathione metabolism (MAPEG) superfamily (Jakobsson et al., 1999). 
With the recent advent of several insect genome sequencing projects, GSTs were identified in the Drosophila, Anopheles, Apis and Bombyx mori as 37, 28, 8 and 23 respectively. The Cystolic GSTs was categorized into seven classes such as Delta, Epsilon, Omega, Theta, Zeta and as well as unclassified GSTs based on the sequence homology, immunological relationships, substrate specifity and inhibitor sensitivity (Chelvanayagam et al., 2001; Ranson et al., 2001, 2002; Ding et al., 2003). Among these classes, epsilon and delta classes present only in insects and additionaly, Alpha, Mu and Pi have been identified in mammalian species (Sheehan et al., 2001).

In insects, several studies showed the significant GST activities towards the detoxification of compounds. In fall armyworm, organophosphate and pyrethroid resistant strains showed the increased GST activities against the model substrates 1-chloro-2,4-dinitro benzene (CDNB) and 1,2-dichloro-4-nitrobenzene (DCNB) (Yu, 1992). In Helicoverpa armigera, phoxim resistant varities exhibit the increased GST level against the OP phoxim (Wu et al., 1997). Also, GST3 of Plutella xylostella and diamondback moth shows more activities toward DCNB and OP (Chiang and Sun, 1993; Ku et al., 1994; Huang et al., 1998).

It's highly essential to understand the metabolic pathways of silkworm related to the degradation of xenobaitics. Hence, we characterized the silkworm cytosolic GSTs, UDP-glucuronosytransferases (UGTs) and carboxyeterases which are the phase I enzymes related to the degradation of xenobaitics. Further, the characterization of these enzymes and the evolutionary analsysis of GSTs were carried out and the functional annotation of these enzymes and their interaction network was created. This study helps to provide preliminary insights into the silkworm Phase I enzymes function against the xenobiotic agents.

\section{Material and Methods:-}

\section{Data resources:}

A total of 23 GST sequences of silkworm were retrieved from the NCBI database. Further, these 23 sequences were classified into six classes such as 4 delta, 8 epsilon, 4 omega, 2 sigma, 1 theta, 2 zeta and 2 unclassified GST (Yu et al., 2008). The xenobiotics biodegradation and metabolism pathway genes of silkworm were analyzed using the information of the Kyoto Encyclopedia of Genes and Genomes (KEGG) pathway database (Kanehisa et al., 2004). The protein-protein interaction data of silkworm were utilized from the SilkPPI database (Sumathy et al., 2014).

\section{Characterization of GSTs:}

The multiple sequence alignment of silkworm GSTs were carried out using the clustal omega program (Sievers et al., 2011). Further, the phylogenetic tree of silkworm GSTs was created using the MEGA6 software (Tamura et al., 2013). The GSTs of silkworm were analyzed for the conserved domain using the HMMER program (Eddy 1998). Further, the motif anlaysis of the silkworm GSTs were carried out using the MEME software (Bailey et al., 2009).

\section{Interaction studies of the pathway:}

The GSTs and their related proteins of xenobiotics biodegradation and metabolism pathway were identified. The identified proteins of the pathway were utilized to study about their interacting proteins using the SilkPPI dataset which was identified using the computational methods. The interaction network of interacting proteins was created and vizualized using the Cytoscape program (Shannon et al., 2003). The functional annotation of these interacting proteins was analysed using Gene ontology information.

\section{Result and Discusion:-}

In cellular detoxification, GSTs plays a vital role by catalyzing the conjugation of glutathione (GSH) with a various endogenous and xenobiotic alkylating agents, including environmental toxins, products of oxidative stress and therapeutic drugs. Hence, it is highly essential to characterize the silkworm GSTs. The GST sequences of silkworm were mapped with the conserved domains and the superfamily. It contains the thioredoxin_like and GST_C_family superfamily along with the GST active site. The GST active site is consists of a specific GSH binding site (G-site) and a nonspecific substrate binding site (H-site), dimer interface, C-terminal domain interface and $\mathrm{N}$-terminal domain interface. Additionally, the Epsilon and Delta type of GSTs contains GST_N_Delta_Epsilon and GST_C_Delta_Epsilon. All GSTs includes G-site while the H-site varies between different classes and isotypes (Aceto et al., 1997; Dragani et. al., 1997; Stenberg et.al., 2000; Cocco et al., 2001). Dimer interface formed by the C-terminal alpha helical domains and N-terminal TRX-fold residue. The N-capping box has a primary role in the formation and stability of $\alpha$-helices (Harper and Rose, 1993; Aurora and Rose, 1998). N-capping box contains a 
reciprocal backbone-side-chain hydrogen bond interaction between the N3 (Glu/Asp) and N-cap (Ser/Thr) and regions.

\section{Motif and phylogenetic analysis:}

The GSTs were analyzed for the motif using the Multiple Entropy for Motif Elicitation and found ten conserved motifs with zero or more occurrences per sequence of GSTs. The pictorial representation of the ten motifs given in the Figure 1. Some of the motifs vary in $\mathrm{N}$ terminal region or $\mathrm{C}$ terminal regions that are considered to be as partial GSTs. Each Conserved motifs are shown in different colors with the E-value. The Multiple sequence alignment of the GSTs was carried out, and the phylogentic tree was constructed using the Neighbor-joining method of MEGA software with 1000 bootstrap value. It was shown that GSTs were grouped according to the type of GSTS, ie. delta, epsilon, omega, sigma, theta, zeta (Figure 2).

\section{Xenobiotics biodegradation and metabolism pathway:}

In silkworm, xenobiotics biodegradation and metabolism pathway play an important role in detoxification of the system. In xenobiotic metabolism, several metabolic enzymes such as GST, cytochrome P450 monooxygenases (P450s), esterases, etc involved in various compounds degradation i.e. styrene, benzoate, toluene, caprolactam, Bisphenol, Aminobenzoate, naphthalene, ethylbenzene, chloroalkane degradation and various xenobiotics. Therefore, all the Xenobiotics biodegradation and metabolism pathway genes of silkworm were studied to understand the mechanisms. Most of the identified genes of silkworm from the Xenobiotics biodegradation and metabolism belong to glutathione S-transferase, glucuronosyltransferase, carboxylesterase, dihydropyrimidine dehydrogenase, dihydrodiol dehydrogenase, carbonyl reductase etc.

\section{Interaction Studies:}

Here, we identified the GST and related genes of xenobiotics metabolic pathways and their interactions with the other proteins were studied using the protein-protein interaction information of silkworm. Previously, the proteinprotein interactions of silkworm were computationally identified using the interlog method with the available model organisms i.e Drosophila, yeast, (Sumathy et al., 2014). Around 128 protein-protein interactions of silworm were maped in this dataset with the criteria of the directerectly interacting pair (Figure 3). The identified interactions were retrieved with their respective interaction score and p-value. In Silkworm PPI dataset, each of the predicted proteinprotein interaction pairs was verified using the microarray data and the Gene annotation information. Hence, interactions were computationally identified using the orthologs information of model organisms that shown the experimentally verified interaction. The predicted protein-protein interactions were visualized using the cytoscape program. In this interaction network, the number of edges represents the degree of a node. Here, 112 nodes were mapped in this network that includes 15 self loops and 4 isolated nodes. This network obeys the power law, i.e. few nodes have more degree and larger nodes have less degree.

\section{Functional annotation:}

Further, the GSTs of silkworm and their interaction proteins were functionally annotated based on the homology similarity with other proteins using Gene Ontology Database [http://www.geneontology.org] and Gene Ontology (GO) terms were assigned to the proteins. The functional annotation was classified into the biological process, cellular component and molecular function (Figure 4). The biological process includes etc. The Functional enrichment analysis was carrired out using Gostat progam that was shown in Table.1. The high level GO terms were mapped namely macromolecular complex, nuclear import, protein import into nucleus, protein transporter activity, intracellular protein transport, protein targeting, nuclear transport, nucleocytoplasmic transport, Protein import, protein transport, the establishment of protein localization, protein localization, macromolecule localization.

\section{Conclusion:-}

In this present study, the silkworm GSTs were characterized and xenobiotics biodegradation and metabolism pathway were well studied. Further, interaction proteins of the pathway were analyzed and vizualized. The functional annotation of the interacting proteins was identified. This study will be helpfull to understand the mechanism beneath the xenobiotics biodegradation and metabolism pathway and the interactions among GSTs with other proteins in mulberry silkworm. 
Figure 1:- Schematic representation of Silkworm GSTs conserved motifs Name ? p-value Motit Location

1. Delta_ 1

$3.57 \mathrm{e}-109$

2. Delta 2

$1.09 \mathrm{e}-110$

3. Delta_3

4. Delta_4

4.41e-109

5. Epsillan_1

$2.05 e-90$

$3.006-122$

6. Epsillan_2

$3.03 e-100$

7. Epsillan 3

4.12e-101

g. Epsillan_4

$1.32 \mathrm{e}-123$

9. Epsillon_5

$0.57 e-119$

10. Epsillon_o

$3.05 e-79$

11. Epsillon_7

$2.300-40$

12. Epsillon_8

$5.34 \mathrm{e}-47$

13. Omega_1

$5.85 e-49$

14. Omega_2

$6.39 e-58$

15. Omega_3

2. $400-41$

16. Omega_4

$9.25 e-53$
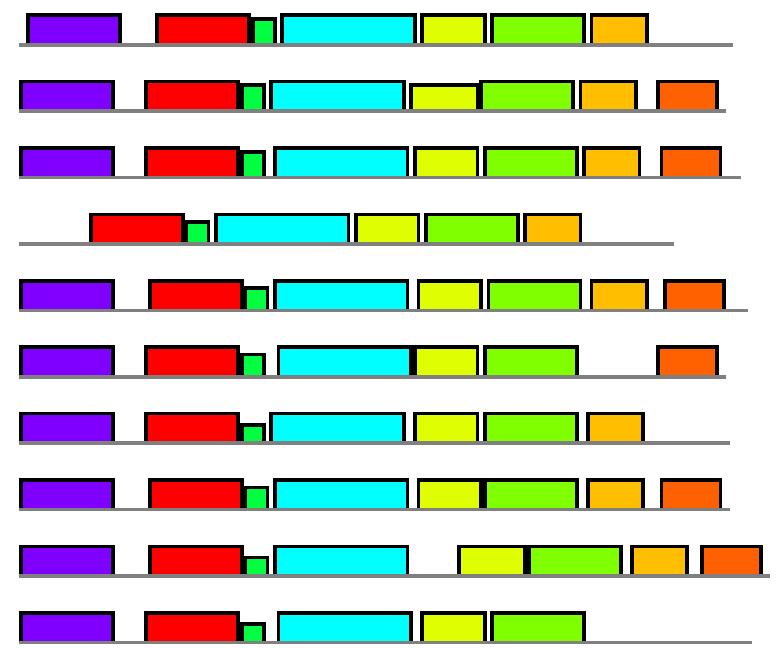

17. Sigma

$0.27 e-76$

18. Sigma_2

$1.83 e-78$

19. Theta_1

$7.50 e-37$

20. Zeta_1

$5.85 e-00$

21. Zeta_2

$2.050-50$

22. Unclassifted_1

$3.300-72$

23. Unclassited 2

1.50 e-94

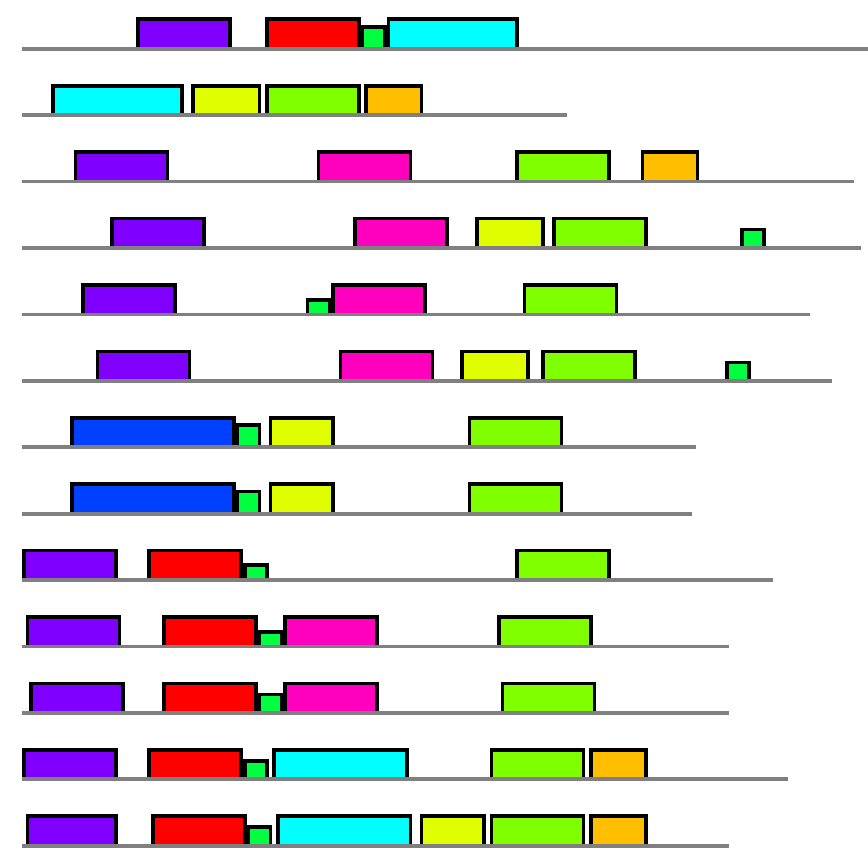


Figure 2:- Phylogenetic analysis of silkworm GSTs.

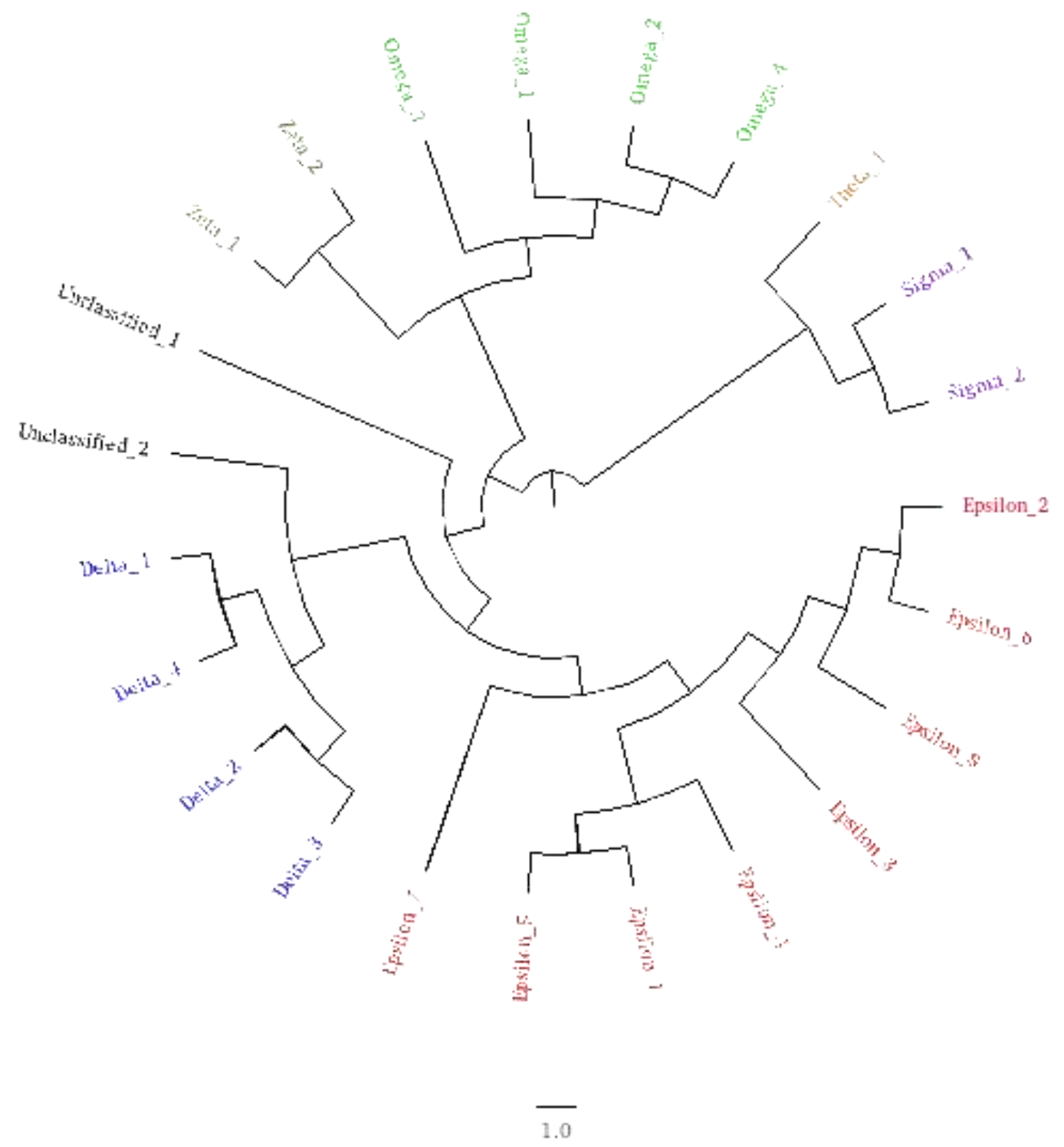


Figure 3:- Interaction network of silkworm GSTs and their interactions proteins.

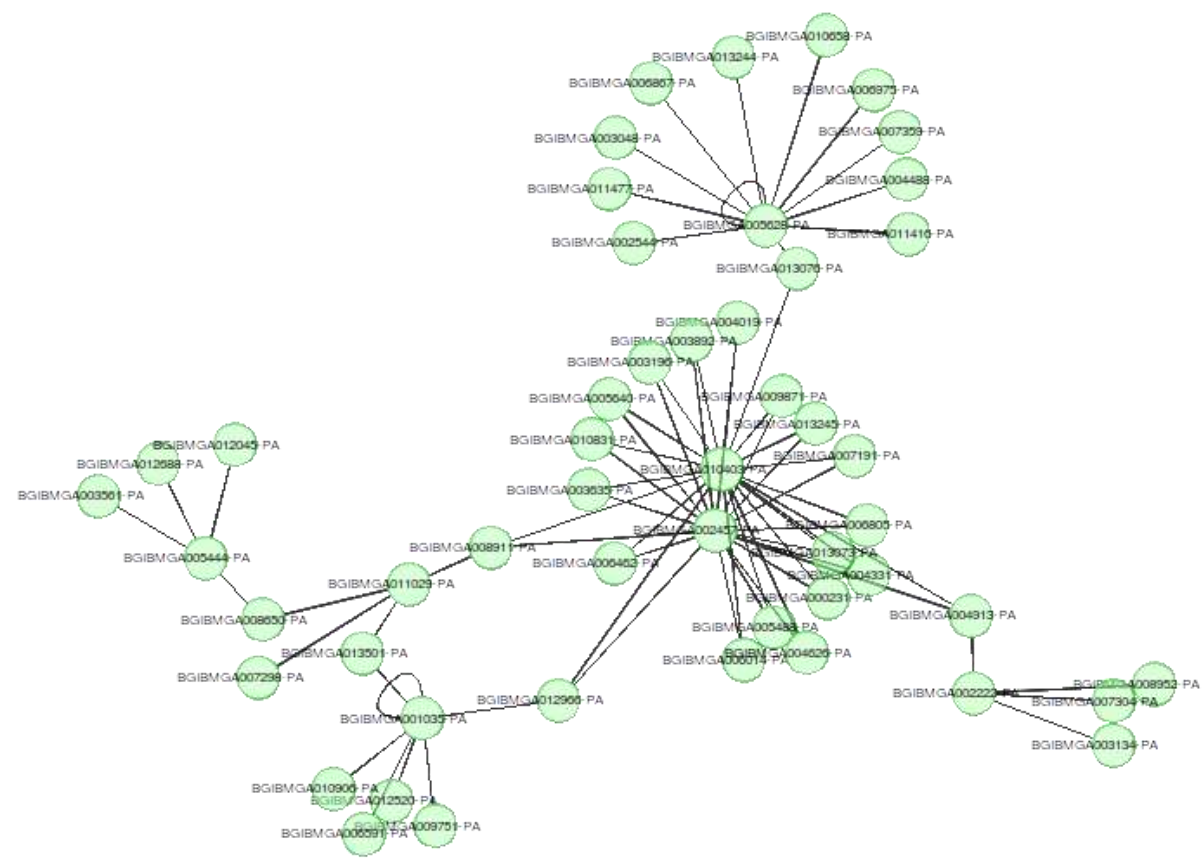

Table 1:- Functional encrichment of GSTs and their interacting proteins

\begin{tabular}{|l|l|l|l|l|}
\hline GO_id & GO_Description & Count & Total count & p-value \\
\hline GO:0032991 & macromolecular complex & 17 & 371 & 0.000191 \\
\hline GO:0051170 & Nuclear import & 4 & 11 & 0.00155 \\
\hline GO:0006606 & protein import into nucleus & 4 & 11 & 0.00155 \\
\hline GO:0008565 & protein transporter activity & 5 & 23 & 0.00155 \\
\hline GO:0006886 & intracellular protein transport & 7 & 62 & 0.00185 \\
\hline GO:0006605 & proteintargeting & 5 & 26 & 0.00185 \\
\hline GO:0051169 & nuclear transport & 4 & 14 & 0.00185 \\
\hline GO:0006913 & nucleocytoplasmic transport & 4 & 14 & 0.00185 \\
\hline GO:0017038 & Protein import & 4 & 17 & 0.00378 \\
\hline GO:0015031 & protein transport & 7 & 79 & 0.00511 \\
\hline GO:0045184 & establishment of protein localization & 7 & 82 & 0.00589 \\
\hline GO:0008104 & protein localization & 7 & 85 & 0.00677 \\
\hline GO:0033036 & macromolecule localization & 7 & 87 & 0.00723 \\
\hline
\end{tabular}


Figure 4:- Functional annotation of silkworm GSTs and their interactions proteins.

\section{Biological process}

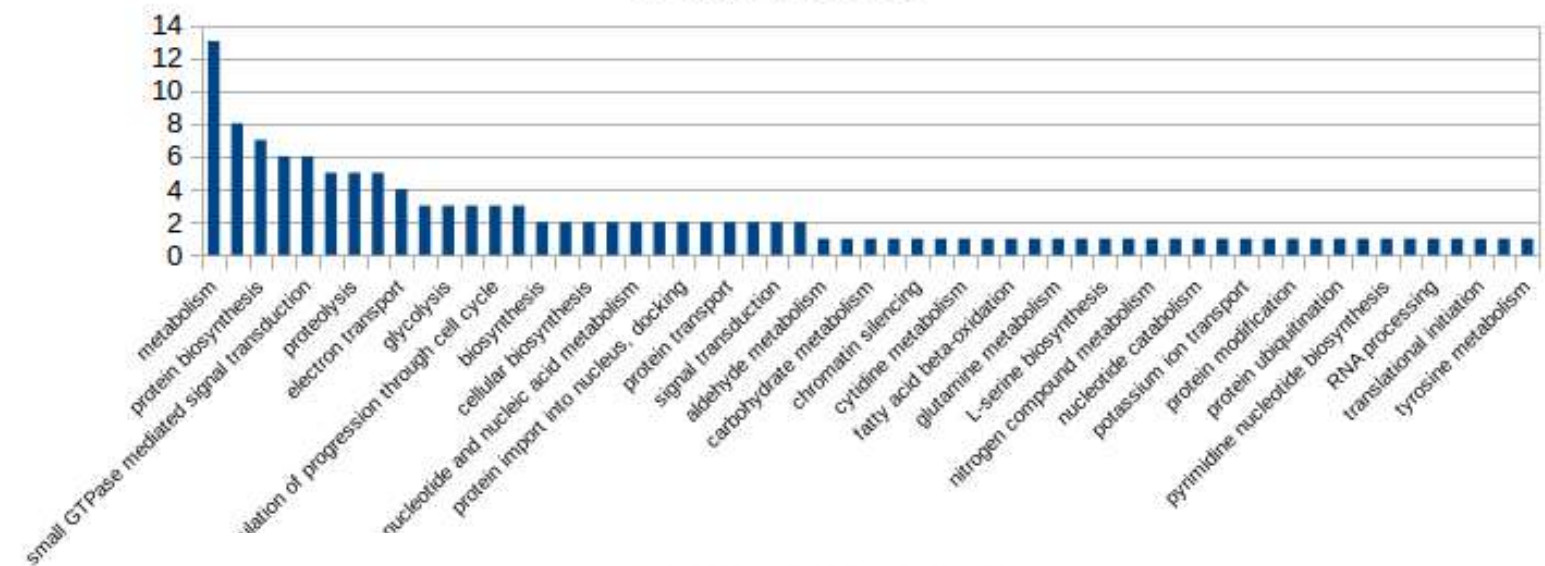

\section{Cellular component}

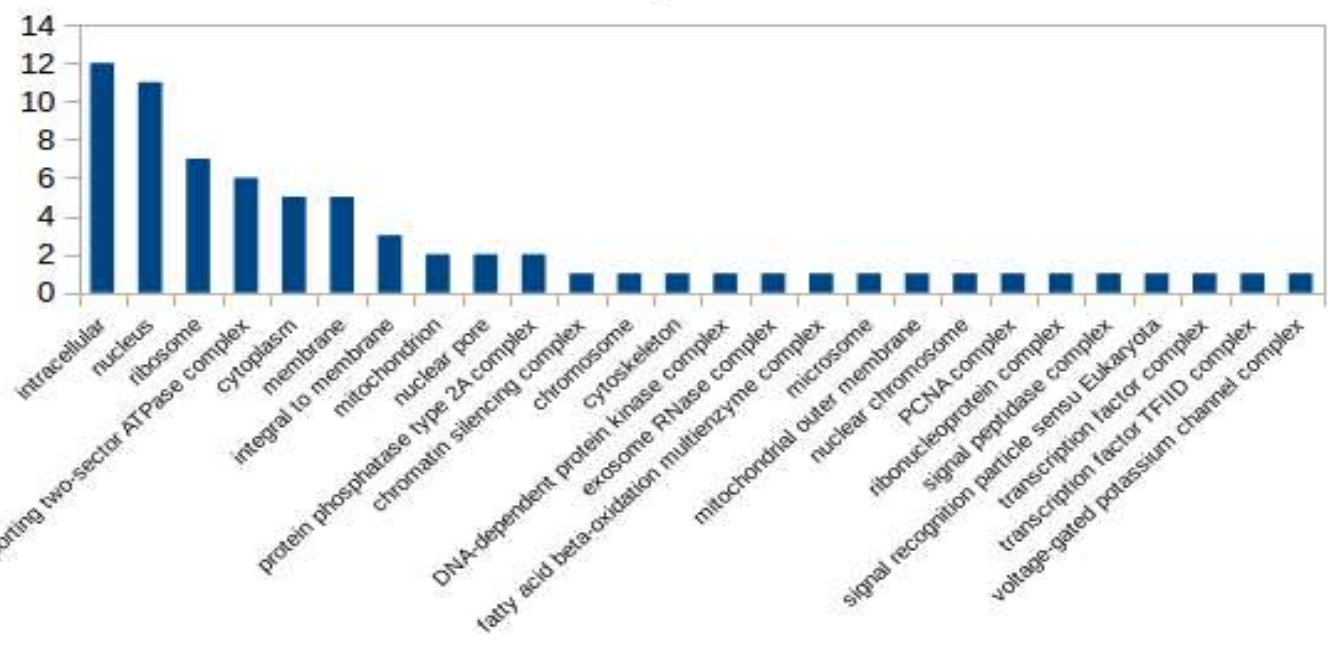

Molecular function

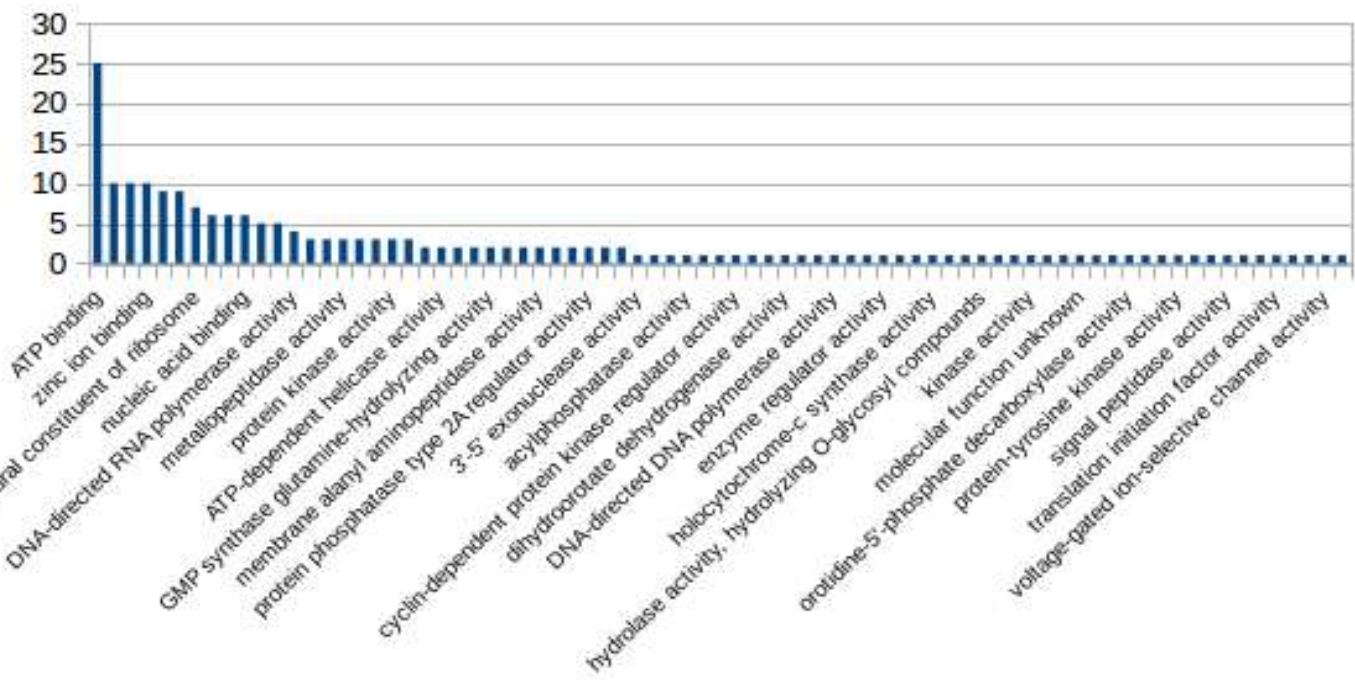




\section{References:-}

1. Aceto A., Dragani B., Melino S., Allocati N., Masulli M., Di Ilio C., Petruzzelli R. Identification of an Ncapping box that affects the $\alpha 6$-helix propensity in glutathione S-transferase superfamily proteins: a role for an invariant aspartic residue. Biochem. J. 1997;322:229-234.

2. Aurora R., Rose G. D. Helix capping.Protein Science.1998;7:21-38.

3. Bailey TL, Boden M, Buske FA, et al (2009) MEME Suite: Tools for motif discovery and searching. Nucleic Acids Res. 37.

4. Chelvanayagam, G., Parker, M.W., Board, P.G., 2001. Fly fishing for GSTs: a unified nomenclature for mammalian and insect glutathione transferases. Chem. Biol. Interact. 133, 256-260.

5. Chiang, F.M., Sun, C.N., 1993. Glutathione transferase isozymes of diamondback moth larvae and their role in the degradation of some organophosphorus insecticides. Pestic. Biochem. Physiol. 45, 7-14.

6. Clark, A.G., Shamaan, N.A., 1984. Evidence that DDT-dehydrochlorinase from the house fly is a glutathione Stransferase. Pestic. Biochem. Physiol. 22, 249-261.

7. Cocco R., Stenberg G., Dragani B., Rossi Principe D., Paludi D., Mannervik B., Aceto A. The folding and stability of human alpha class glutathione transferase A1-1 depend on distinct roles of a conserved N-capping box and hydrophobic staple motif. J. Biol. Chem. 2001;276:32177-32183.

8. Ding, Y., Ortelli, F., Rossiter, L.C., Hemingway, J., Ranson, H., 2003. The Anopheles gambiae glutathione transferase supergene family: annotation, phylogeny and expression profiles. BMC Genomics 4, 35.

9. Dragani B., Stenberg G., Melino S., Petruzzelli R., Mannervik B., Aceto A. The conserved N-capping box in the hydrophobic core of glutathione S-transferase P1-1 is essential for refolding. Identification of a buried and conserved hydrogen bond important for protein stability. J. Biol. Chem. 1997;272:25518-25523.

10. Eddy SR (1998) Profile hidden Markov models. Bioinformatics 14:755-63.

11. Harper E. T., Rose G. D. Helix stop signals in proteins and peptides: the capping box. Biochemistry.1993;32:7605-7609.

12. Hayes, J.D., Flanagan, J.U., Jowsey, I.R., 2005. Glutathione transferases. Annu. Rev. Pharmacol. Toxicol. 45, 51-88.

13. Huang, H.S., Hu, N.T., Yao, Y.E., Wu, C.Y., Chiang, S.W., Sun, C.N., 1998. Molecular cloning and heterologous expression of a glutathione $\mathrm{S}$-transferase involved in insecticide resistance from the diamondback moth, Plutella xylostella. Insect Biochem. Mol. Biol. 28, 651-658.

14. Jakobsson, P.J., Morgenstern, R., Mancini, J., Ford-Hutchinson, A., Persson, B., 1999. Common structural features of MAPEG-a widespread superfamily of membrane associated proteins with highly divergent functions in eicosanoid and glutathione metabolism. Protein Sci. 8, 689-692.

15. Kanehisa M, Goto S, Kawashima S, Okuno Y, Hattori M (2004) The KEGG resource for deciphering the genome. Nucleic Acids Res 32(suppl 1):D277-D280.

16. Ku, C.C., Chiang, F.M., Hsin, C.Y., Yao, Y.E., Sun, C.N., 1994. Glutathione transferase isozymes involved in insecticide resistance of diamondback moth larvae. Pestic. Biochem. Physiol. 50, 191-197.

17. Lander, J.E., Parsons, J.F., Rife, C.L., Gilliland, G.L., Armstrong, R.N., 2004. Parallel evolutionary pathways for glutathione transferases: structure and mechanism of the mitochondrial class Kappa enzyme rGSTK1-1. Biochemistry 43, 352-361.

18. Morel, F., Rauch, C., Petit, E., Piton, A., Theret, N., Coles, B., Guillouzo, A., 2004. Gene and protein characterization of the human glutathione S-transferase kappa and evidence for a peroxisomal localization. J. Biol. Chem. 279, 16246-16253.

19. Motoyama, N., Dauterman, W.C., 1980. Glutathione S-transferases: their role in the metabolism of organophosphorus insecticides. Rev. Biochem. Toxicol. 2, 49-69.

20. Ranson, H., Claudianos, C., Ortelli, F., Abgrall, C., Hemingway, J., Sharakhova, M.V., Unger, M.F., Collins, F.H., Feyereisen, R., 2002. Evolution of supergene families associated with insecticide resistance. Science 298, 179-181.

21. Ranson, H., Rossiter, L., Ortelli, F., Jensen, B., Wang, X., Roth, C.W., Collins, F.H., Hemingway, J., 2001. Identification of a novel class of insect glutathione S-transferases involved in DDT resistance in the malaria vector, Anopheles gambiae. Biochem. J. 359, 295-304.

22. Shannon P, et al. Cytoscape: A Software Environment for Integrated Models of Biomolecular Interaction Networks Genome Res. 2003;13:2498.

23. Sheehan, D., Meade, G., Foley, V.M., Dowd, C.A., 2001. Structure, function and evolution of glutathione transferases: implications for classification of non-mammalian members of an ancient enzyme superfamily. Biochem. J. 360, 1-16. 
24. Sievers F, Wilm A, Dineen D, et al (2011) Fast, scalable generation of high-quality protein multiple sequence alignments using Clustal Omega. Mol Syst Biol 7:539 . doi: 10.1038/msb.2011.75.

25. Singh, S.P., Coronella, J.A., Benes, H., Cochrane, B.J., Zimniak, P., 2001. Catalytic function of Drosophila melanogaster glutathione S-transferase DmGSTS1-1(GST-2) in conjugation of lipid peroxidation end products. Eur. J. Biochem. 268, 2912-2923.

26. Stenberg G., Dragani B., Cocco R., Mannervik B., Aceto A. A conserved 'hydrophobic staple motif' plays a crucial role in the refolding of human glutathione transferase P1-1. J. Biol. Chem. 2000;275:10421-10428.

27. Sumathy R., Rao A.S., Chandrakanth N. et al. (2014) In silico identification of protein-protein interactions in Silkworm, Bombyx mori. Bioinformation, 10, 56-62.

28. Tamura K, Stecher G, Peterson D, et al (2013) MEGA6: Molecular Evolutionary Genetics Analysis version 6.0. Mol Biol Evol 30:2725-9 . doi: 10.1093/molbev/mst197.

29. Vontas, J.G., Small, G.J., Hemingway, J., 2001. Glutathione S-transferases as antioxidant defence agents confer pyrethroid resistance in Nilaparvata lugens. Bio-chem. J. 357, 65-72.

30. Wu, K., Liang, G., Guo, Y., 1997. Phoxim resistance in Helicoverpa armigera (Lepidoptera: Noctuidae) in China. J. Econ. Entomol. 90, 868-872.

31. Yu, S.J., 1992. Detection and biochemical characterization of insecticide resistance in fall armyworm (Lepidoprera: Noctuidae). J. Econ. Entomol. 85, 675-682.

32. Yu, Q., Lu, C., Li, B., Fang, S., Zuo, W., Dai, F., Zhang, Z., Xiang, Z., 2008. Identification, genomic organization and expression pattern of glutathione S-transferase in the silkworm, Bombyx mori. Insect Biochem Mol Biol. 38, 1158-64. 